\title{
Design and Analysis of Optimization Methods for Subdivision Surface Fitting
}

\author{
Kin-Shing D. Cheng, Member, IEEE, Wenping Wang, Hong Qin, Member, IEEE, \\ Kwan-Yee K. Wong, Member, IEEE, Huaiping Yang, and Yang Liu
}

\begin{abstract}
We present a complete framework for computing a subdivision surface to approximate unorganized point sample data, which is a separable nonlinear least squares problem. We study the convergence and stability of three geometrically motivated optimization schemes and reveal their intrinsic relations with standard methods for constrained nonlinear optimization. A commonly used method in graphics, called point distance minimization, is shown to use a variant of the gradient descent step and thus has only linear convergence. The second method, called tangent distance minimization, which is well known in computer vision, is shown to use the Gauss-Newton step and, thus, demonstrates near-quadratic convergence for zero residual problems but may not converge otherwise. Finally, we show that an optimization scheme called squared distance minimization, recently proposed by Pottmann et al., can be derived from the Newton method. Hence, with proper regularization, tangent distance minimization and squared distance minimization are more efficient than point distance minimization. We also investigate the effects of two step-size control methods—Levenberg-Marquardt regularization and the Armijo rule-on the convergence stability and efficiency of the above optimization schemes.
\end{abstract}

Index Terms-Subdivision surface, fitting, optimization, squared distance.

\section{INTRODUCTION}

SHAPES represented by 3D unorganized geometric points Sare now readily available as the widespread use of 3D scanning devices for shape acquisition becomes a common practice. For geometric processing, we often need to fit a surface to such point samples. Subdivision surface is a preferred representation because of its compactness and ability to accommodate general control mesh connectivity. From the optimization point of view, the surface fitting problem is a separable nonlinear least squares problem. In principle, we need to minimize an objective function consisting of a geometric error term and a smoothing term. The geometric error term can be approximated by different functions measuring the squared distance (SD) between a fitting surface and the target shape. These error functions lead to different local quadratic models of the objective function and result in different optimization efficiency.

\subsection{Our Work}

This paper is an extension to our work [1] presented in the Proceedings of the 12th Pacific Conference on Computer Graphics and Applications (PG '04). We present a general and complete framework for computing a subdivision surface via geometric point fitting. Specifically, we focus on the convergence analysis of optimization schemes for solving

- K.-S.D. Cheng, W. Wang, K.-Y.K. Wong, and Y. Liu are with the Department of Computer Science, The University of Hong Kong, Pokfulam Road, Hong Kong. E-mail: \{ksdcheng, wenping, kykwong, yliu\}@cs.hku.hk.

- H. Qin is with the Department of Computer Science, State University of New York at Stony Brook, Stony Brook, NY 11794-4400. E-mail:qin@cs.sunysb.edu.

- H. Yang is with the Institute of Applied Geometry, Johannes Kepler University, Altenberger Str. 69, 4040 Linz, Austria.

E-mail: hpyang@cs.hku.hk.

Manuscript received 27 May 2006; revised 28 Oct. 2006; accepted 24 Jan. 2007; published online 5 Mar. 2007.

For information on obtaining reprints of this article, please send e-mail to: tvcg@computer.org, and reference IEEECS Log Number TVCG-0073-0506.

Digital Object Identifier no. 10.1109/TVCG.2007.1064.

1077-2626/07/\$25.00 @ 2007 IEEE this surface fitting problem. Suppose that the shape to be fitted, called the target shape, is defined by unorganized data points. To start the fitting process, an initial subdivision surface is first generated from the point cloud by applying the dual marching cubes method [2]. The control points are then optimized by minimizing an objective function through iterative quadratic minimization. New control points are added progressively in order to better capture the features of the target shape; this gives rise to a multistaged optimization problem. Although we use Loop's subdivision surface [3] to handle triangular meshes, the proposed algorithmic flow can naturally apply to other types of subdivision surfaces based on linear schemes.

We consider three geometrically motivated methods in this paper. The first method is based on a point-to-point distance error metric, thus called point distance minimization (PDM) [4]. This method has been used predominantly for decades in graphics and CAD/CAM for curve and surface fitting. It has a monotonic descent but converges slowly. The second method uses a point-to-tangent distance error metric, thus called tangent distance minimization (TDM). This has been used in the computer vision field for model registration [5]. In general, it converges much faster than PDM but is unstable for a target shape with sharp features. The third method, called squared distance minimization (SDM), has been recently proposed by Pottmann and Leopoldseder [6] for B-spline curve and surface fitting.

Our contribution is the systematic study on the convergence behaviors of the above optimization methods in the setting of subdivision surface fitting. The derivations of these methods in the literature are only based on geometric arguments. We establish their equivalences to three wellunderstood optimization methods-steepest descent, the Gauss-Newton method, and the Newton method, respectively - for nonlinear constrained optimization.

This connection to well-known optimization techniques helps explain or understand the practical behaviors of these 
three methods in surface fitting. The steepest descent method is well known to have a linear convergence rate, thus explaining the slow convergence of PDM. The GaussNewton method has quadratic convergence for zero residual problems, but it converges only linearly or may not converge at all for problems with a large residue-this also conforms with the observed behavior of TDM. SDM is a simple geometric incarnation of the Newton method, thus explaining its observed superior convergence in the general case. Based on this interpretation, we apply the LevenbergMarquardt (LM) regularization to TDM and SDM to ensure a monotonic decrease of the objective function, thus improving the robustness of their convergence. Details about these basic optimization techniques can be found in standard texts on optimization (for example, [7], [8]).

\subsection{Related Work}

The problem of computing a compact surface representation of a target shape defined by unorganized data points has many applications in computer graphics, CAD, and computer vision. Numerous approaches have been proposed over the past decades. These include fitting methods based on optimization [9], [10], [11], [12], [13], [14], active surface methods [15], [16], [17], and other approaches [18], [19], [20], [21]. Among parametric surfaces, subdivision surface has gained popularity because of its ability to deal with general object topology, as well as arbitrary connectivity of the control mesh [22]. Some works are closely related to the problem addressed in this paper. In Hoppe et al.'s method [9], a mesh surface is computed to fit unorganized points via optimization of an energy function. In [11], [12], a (noniterative) linear least squares problem is solved to produce a fitting surface composed of B-spline surfaces for quadrangle patches and Catmull-Clark surfaces for extraordinary corner patches. In [13], a Loop's subdivision surface is computed to fit a mesh surface by iterative quadratic optimization, using a simplification of the input mesh as the initial control mesh. All these methods use the point distance (PD) error function to approximate the geometric error between the fitting surface and the target shape in each iteration. This is essentially the parameter correction method by Hoschek [4], but we will refer to this scheme as PDM. PDM belongs to the category of the alternating method [23], [7] for solving separable nonlinear least squares problems; it has only a linear convergence rate and converges slowly in practice, as we will demonstrate later in this paper.

TDM uses another error function, called the tangent distance (TD) error term, based on a point-to-tangent distance, and it has been used in computer vision for 3D model registration by Chen and Medioni [5] and active curve fitting by Blake and Isard [15]. In the extension to their work in [13], Marinov and Kobbelt apply a combination of the PD error term and TD error term to improve the efficiency of their surface fitting method [24], without considering the issues of convergence analysis and step-size control.

SDM uses the so-called squared distance (SD) error term, which is first considered in [25] and further investigated in detail from a geometric point of view by Pottmann and Hofer [26] with applications to shape fitting with B-spline curves and surfaces [6], [27]. However, they did not give a convergence analysis or consider step-size control for ensuring convergence.

In this work, we emphasize the convergence behaviors of PDM, TDM, and SDM in the setting of subdivision surface fitting from an optimization point of view and investigate the step-size control schemes as applied to these methods. Our results provide new and useful insights to the practical surface fitting procedures used in computer graphics. A variant of the SDM method, along with TDM and PDM, has been recently studied in [28]. The connections of our work here to that of [28], as well as their differences, are elaborated in Section 5.

\section{Fitting Algorithm}

\subsection{Problem Formulation}

Suppose that the input data points, defining a target shape $\Gamma$, are sampled from an underlying target surface $\Gamma_{T}$, which is a manifold surface of an arbitrary genus. For the convergence analysis, the second order differentiability of $\Gamma_{T}$ is assumed. Our goal is to reconstruct the surface $\Gamma_{T}$ by computing a subdivision surface from $\Gamma$.

Let $P(s, t)$ be a local parameterization of a fitting surface $\mathcal{S}$. The fitting error between $\mathcal{S}$ and the target surface $\Gamma_{T}$ is measured by the sum of SDs from a set of dense sample points on $\mathcal{S}$ to $\Gamma_{T}$. Denote these sample points by $S_{k}=P\left(s_{k}, t_{k}\right)$, which are linear combinations of the control points $P_{i}, k=1,2 \ldots, m$, of a Loop's subdivision surface $\mathcal{S}$. We assume that $m$ is much greater than $n$, the number of control points, so that the fitting problem is properly constrained. Let $V_{k}=V\left(u_{k}, v_{k}\right) \in \Gamma_{T}$ be the closest point from the sample point $S_{k}$ on the fitting $\mathcal{S}$ to the target surface $\Gamma_{T}$. The fitting error at $S_{k}$ is then given by $f_{k}=\left\|S_{k}-V\left(u_{k}, v_{k}\right)\right\|$. The point $V\left(u_{k}, v_{k}\right) \in \Gamma_{T}$ is called the foot point of $S_{k}$.

Denote $\mathcal{P}=\left\{P_{i}\right\}_{i=1}^{n}$ and $\mathcal{U}=\left\{\left(u_{k}, v_{k}\right)\right\}_{k=1}^{m}$. The control points $\mathcal{P}$ of the best fitting surface $\mathcal{S}$ are computed by solving the following optimization problem:

$$
\min F(\mathcal{P}, \mathcal{U})=F_{e}(\mathcal{P}, \mathcal{U})+F_{s}(\mathcal{P}),
$$

where $F_{e}=\frac{1}{2} \sum_{k=1}^{m} f_{k}^{2}=\frac{1}{2} \sum_{k=1}^{m}\left\|S_{k}-V\left(u_{k}, v_{k}\right)\right\|^{2}$ is the $L_{2}$ fitting error, and $F_{s}(\mathcal{P})$ is a regularization term that is a quadratic function of the control points $\mathcal{P}$. The variables in the function $F$ are the control points $\mathcal{P}=\left\{P_{i}\right\}$ and the parameter values $\mathcal{U}=\left\{\left(u_{k}, v_{k}\right)\right\}$. Clearly, $F$ is quadratic in $\mathcal{P}$ but is, in general, a highly nonlinear function of $\mathcal{U}$.

We may treat $\mathcal{P}$ as basic variables and $\mathcal{U}$ as dependent variables, since it is required that $V\left(u_{k}, v_{k}\right)$ be the foot point of the sample point $S_{k}$, which is a linear function of $\mathcal{P}$. This leads to the following commonly used optimization strategy in surface fitting: Given an initial fitting surface with the sample points $S_{k}$, one first computes that foot points $V\left(u_{k}, v_{k}\right)$. With $\mathcal{U}$ being known, one updates the control points $\mathcal{P}$ by minimizing $F(\mathcal{P}, \mathcal{U})$ over the control points $\mathcal{P}$; this is done by solving a linear system of equation, since $F(\mathcal{P}, \mathcal{U})$ is quadratic in $\mathcal{P}$, and $\mathcal{U}$ has been fixed. The above two steps of foot-point computation and control-point computation are iterated to further improve the fitting error until convergence. 
This commonly used iterative fitting method is called the PDM method. Because of the separate treatments of variables $\mathcal{P}$ and $\mathcal{U}$, the optimization problem defined in (1) is also called a separable variable problem. We will also consider two other more efficient local optimization schemes based on the same framework, that is, TDM and SDM.

An initial fitting surface is needed to start the above iterative procedure. It is natural to begin with an initial fitting surface having the same topology as the target shape and a simple control mesh. Control points need to be inserted progressively as optimization proceeds to make the fitting surface better capture the fine features of the target shape. This means that we need to consider a multistaged optimization problem with proper scheduling of adding new control points.

\subsection{Algorithmic Flow}

Our proposed fitting procedure has the following main steps:

1. Normalization. Normalize the target shape by uniform scaling to fit it within the cube $[0,1]^{3}$.

2. Precomputation. Precompute the distance field, as well as the tangential and curvature information of the target surface $\Gamma$ for setting up error terms.

3. Initial mesh. Compute an initial control mesh using the dual marching cubes method [2].

4. Points sampling. Generate $m$ dense sample points $S_{k}^{0}$ on the current fitting surface using the method in [29], [30].

5. Error function setup. Use the sample points generated in Step 4 to set up the error function:

$$
F_{L}(\mathcal{P})=\frac{1}{m} \sum_{k=1}^{m} F_{L, k}(\mathcal{P})+F_{s}(\mathcal{P}),
$$

where $F_{L, k}(\mathcal{P})$ is one of the three error terms to be introduced in Section 2.4.

6. Minimization. Update the control points by minimizing the quadratic function $F_{L}(\mathcal{P})$. This is done by solving a linear system of equations using the conjugate gradient (CG) method.

7. Error evaluation. Sample new points $S_{k}^{1}$ on the fitting surface with updated control points, and compute their foot points $V_{k}^{1}$. Next, compute the maximum error $E_{m}$ and the root-mean-square (RMS) error $E_{r m s}$, where

$$
E_{m}=\max _{k}\left\{\left\|S_{k}^{1}-V_{k}^{1}\right\|_{2}\right\}
$$

and

$$
E_{r m s}=\left[\frac{1}{m} \sum_{k}\left\|S_{k}^{1}-V_{k}^{1}\right\|_{2}^{2}\right]^{\frac{1}{2}} .
$$

Here, $\left\|S_{k}^{1}-V_{k}^{1}\right\|_{2}$ is called the local error. The algorithm is terminated if $E_{m}$ or $E_{r m s}$ falls below a prespecified error threshold or the number of iterations reaches some limit. If the fitting error has been reduced significantly in this step by the current iteration (but still larger than the threshold), then go to Step 4 to start the next iteration. Otherwise, go to Step 8.

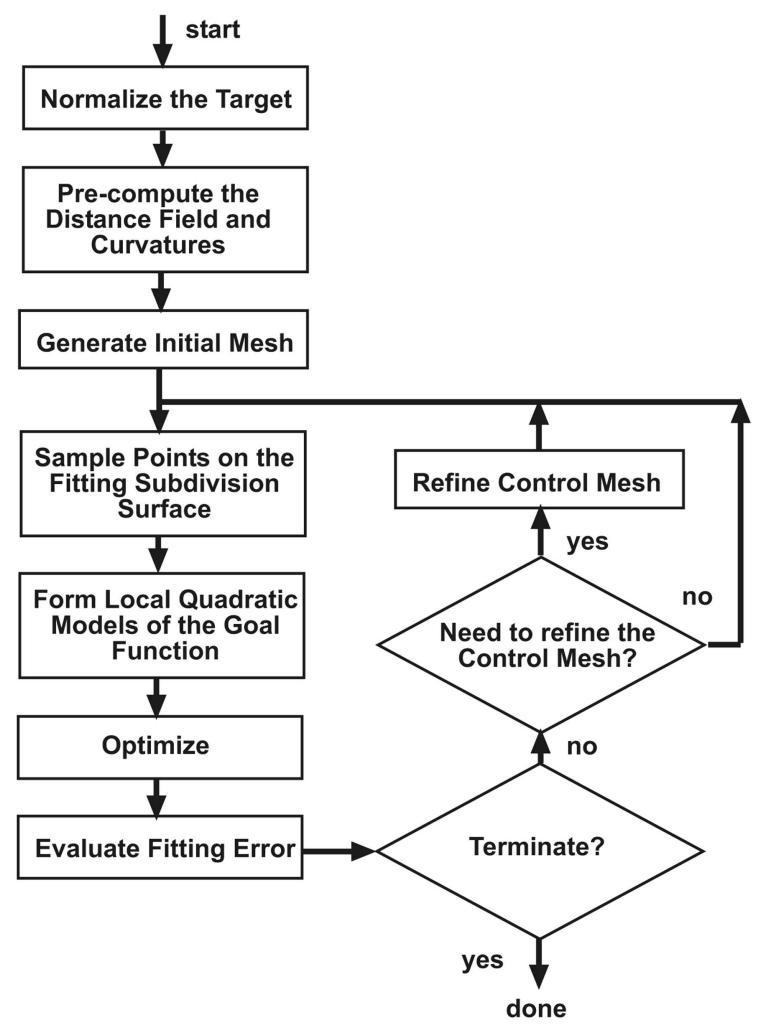

Fig. 1. Workflow.

8. Refinement. New control points are inserted in the regions of large fitting error $E_{m}$. Go to Step 4 for the next iteration.

The flowchart is shown in Fig. 1. Note that remeshing could be used in Step 3 to reduce the number of extraordinary vertices whose valences are not six.

\subsection{Preprocessing and Initialization}

To quickly obtain the foot points $V_{k}^{0}$ of the sample points $S_{k}^{0}$, we precompute an adaptive distance field of the target shape using the idea in [31]. The precomputed information such as distances and foot points are stored at the corners of adaptive octree cells. During the optimization process, the foot point of a sample point $S_{k}^{0}$ is computed by trilinear interpolation from the stored values at the corners of the smallest cell containing $S_{k}^{0}$.

The normal vector and the principal curvatures of the target surface $\Gamma_{T}$ at $V_{k}$ are also precomputed from the point cloud $\Gamma$ for setting up the TD error functions and the SD error functions. For a given target point $V_{k}$, we first find its neighboring points $V_{k, j}$. A local coordinate frame at $V_{k}$ can then be defined by the principal curvature directions and the normal direction that are the eigenvectors of the covariance matrix $\mathcal{C} \mathcal{V}$ given by

$$
\mathcal{C V}=\sum_{j}\left(V_{k, j}-V_{k, c}\right)\left(V_{k, j}-V_{k, c}\right)^{T}
$$

where $V_{k, c}$ is the centroid of $V_{k}$ 's neighboring points. A polynomial $z=k_{1} x^{2}+k_{2} y^{2}$ is then fitted to the neighboring points $V_{n(i, j)}$ in this local frame, and the principal curvatures are simply set to $2 k_{1}$ and $2 k_{2}$. 


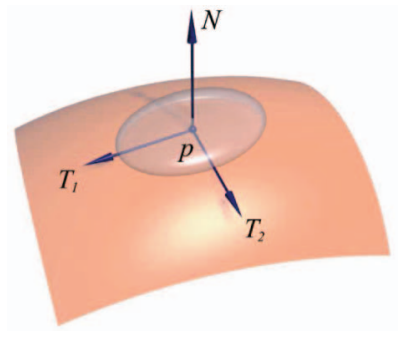

Fig. 2. An isosurface of the SD error term.

We use the dual marching cubes method devised by Schaefer and Warren [2] to compute the initial control mesh from the dual grid of cells of an adaptive octree partition of the target data set. The meshes generated by this method are topologically faithful, adaptive to curvature or features of the target shape and, therefore, more desirable than those generated by the ordinary marching cubes method [32], which tends to produce too many fragmented triangles, even in flat regions of the target shape, as observed in [1].

\subsection{Error Functions}

\subsubsection{PD Error Term}

Suppose that the fitting surface has been sampled by the points $S_{k}^{0}$, whose foot points are $V_{k}^{0}$. Let $S_{k}$ be the variable points of $S_{k}^{0}$, depending on the variable control points $\mathcal{P}$. A simple error term is given by

$$
f_{P D, k}^{2}=\left\|S_{k}-V_{k}^{0}\right\|^{2},
$$

which is called the PD error term because $f_{P D, k}$ is the distance between $S_{k}$ and $V_{k}^{0}$. The optimization scheme resulting from using the PD error term for $F_{L, k}(\mathcal{P})$ in (2) is called PDM.

\subsubsection{TD Error Term}

When the target surface $\Gamma_{T}$ is relatively flat around $V_{k}^{0}$, the tangent plane of $\Gamma_{T}$ at $V_{k}^{0}$ is a good approximation to $\Gamma_{T}$ in a neighborhood of $V_{k}^{0}$. This observation leads to the so-called TD error term, defined as

$$
f_{T D, k}^{2}=\left|\left(S_{k}-V_{k}^{0}\right)^{T} N_{k}\right|^{2},
$$

where $N_{k}$ is a constant unit normal vector to $\Gamma$ at $V_{k}^{0}$. Clearly, $f_{T D, k}^{2}$ is the SD from $S_{k}$ to the tangent plane $\left(X-V_{k}^{0}\right) N_{k}=0$. The optimization scheme resulting from using the TD error term is called TDM.

\subsubsection{SD Error Term}

Now, we consider the second-order approximation to the SD from $S_{k}$ to $\Gamma_{T}$, as proposed in [6], [25]. Let $d$ be the signed distance such that $|d|=\left\|S_{k}^{0}-V_{k}^{0}\right\|$. Let $\rho_{1}$ and $\rho_{2}$ denote the principal curvature radii of $\Gamma_{T}$ at $V_{k}^{0}$ associated with the principal unit direction vectors $T_{1}$ and $T_{2}$. We make the following convention on the signs of $d$ and $\rho_{i}, i=1,2$. Suppose that $N$ is a normal vector to the target surface $\Gamma_{T}$. If the sample point $S_{k}$ is on the side of $\Gamma_{T}$ pointed to by $N$, that is, $N \cdot\left(S_{k}^{0}-V_{k}^{0}\right)>0$, then $d>0$; otherwise, $d \leq 0$. Similarly, if the curvature center in the normal section along the tangent vector $T_{i}$ is on the side of $\Gamma_{T}$ pointed to by $N$, then $\rho_{i}>0$; otherwise, $\rho_{i} \leq 0, i=1,2$.

Since $V_{k}^{0}$ is locally the closest point from $S_{k}^{0}$ to $\Gamma_{T}$, then we have $|d|<\left|\rho_{i}\right|$ when $d$ and $\rho_{i}$ have the same sign. It can
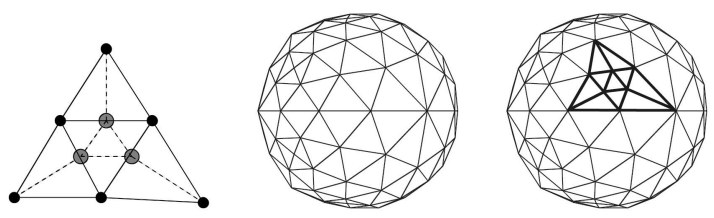

Fig. 3. Triangle split.

be shown [25], [26] that the second-order approximation to the true SD is

$$
\begin{aligned}
\hat{f}_{S D, k}^{2}= & \alpha_{1}\left[\left(S_{k}-V_{k}^{0}\right)^{T} T_{1}\right]^{2}+\alpha_{2}\left[\left(S_{k}-V_{k}^{0}\right)^{T} T_{2}\right]^{2} \\
& +\left[\left(S_{k}-V_{k}^{0}\right)^{T} N\right]^{2},
\end{aligned}
$$

where $\alpha_{1}=d /\left(d-\rho_{1}\right)$, and $\alpha_{2}=d /\left(d-\rho_{2}\right)$. The coefficient $\alpha_{1}$ (or $\alpha_{2}$ ) becomes negative if $\rho_{1}$ (or $\rho_{2}$ ) and $d$ have the same sign. Denote $[\alpha]_{+}=\max \{\alpha, 0\}$. To have a nonnegative error function, $\hat{f}_{S D, k}^{2}$ is modified to

$$
\begin{aligned}
f_{S D, k}^{2}= & {\left[\alpha_{1}\right]_{+}\left[\left(S_{k}-V_{k}^{0}\right)^{T} T_{1}\right]^{2}+\left[\alpha_{2}\right]_{+}\left[\left(S_{k}-V_{k}^{0}\right)^{T} T_{2}\right]^{2} } \\
& +\left[\left(S_{k}-V_{k}^{0}\right)^{T} N\right]^{2} .
\end{aligned}
$$

This term $f_{S D, k}^{2}$ is called the $S D$ error term, since it is derived from a second-order approximation to the true SD. The ellipsoid in Fig. 2 shows an isodistance surface of the SD error term for the case $\alpha_{1}>0$ and $\alpha_{2}>0$; the SD error term reduces to the TD error term if $\alpha_{1}=0$ and $\alpha_{2}=0$. The optimization scheme resulting from using the SD error term is called SDM.

\subsection{Smoothing Term}

The smoothing term in (2) is defined as

$$
F_{s}=\frac{\lambda}{n} \sum_{i=1}^{n} W\left(P_{i}\right)^{T} W\left(P_{i}\right),
$$

where $P_{i}, i=1,2, \ldots, n$, are the control points, and $W(\cdot)$ is a discrete version of Laplacian [33]. It is difficult to automatically choose an appropriate value of the coefficient $\lambda$ [34]; therefore, most existing methods choose $\lambda$ in a heuristic manner. Likewise, in our test examples, the initial value for $\lambda$ is set to 0.001 at the beginning and is reduced gradually as the optimization proceeds.

We use the CG method to minimize the error function (2). This iterative solver is terminated if the relative error improvement is less than $10^{-6}$ or the number of iterations reaches 200 . These parameters produce satisfactory results in our experiments.

\subsection{Local Refinement}

When the fitting error remains large due to the insufficient number of control points, new control points are inserted to triangles that have large local errors and split the triangles in a one-to-four manner (see Fig. 3). This step is called local refinement. To avoid undesirable T-vertices, the neighboring triangles are also split, following the Red-Green splitting scheme [35].

\section{Optimization Properties}

In this section, we shall establish the connection between the three optimization techniques introduced so far-PDM, TDM, and SDM — and the standard optimization techniques 
in optimization theory. Due to the space limit, we shall only discuss some basic facts that we are going to use directly; the reader is referred to standard texts (for example, [7], [8]) for a detailed introduction to optimization theory.

\subsection{Basics of Optimization}

First, consider the Newton method. Given an objective function $f(x): \mathcal{R}^{N} \rightarrow \mathcal{R}$ and the current variable value $x_{c}$ near a local minimum of $f(x)$, the next iterate $x_{+}$is the minimizer of the local quadratic model $m_{c}(x)$ of $f(x)$ about $x_{c}$, where

$$
\begin{aligned}
m_{c}(x)= & f\left(x_{c}\right)+\nabla f\left(x_{c}\right)^{T}\left(x-x_{c}\right) \\
& +\frac{1}{2}\left(x-x_{c}\right)^{T} \nabla^{2} f\left(x_{c}\right)\left(x-x_{c}\right),
\end{aligned}
$$

which is the second-order Taylor expansion of $f(x)$ at $x_{c}$. If $\nabla^{2} f\left(x_{c}\right)$ is positive definite, $x_{+}$is the unique solution of the equation:

$$
0=\nabla m_{c}\left(x_{+}\right)=\nabla f\left(x_{c}\right)+\nabla^{2} f\left(x_{c}\right)\left(x_{+}-x_{c}\right) .
$$

The Newton method has local quadratic convergence.

Various iterative schemes can be obtained by replacing the Hessian $\nabla^{2} f\left(x_{c}\right)$ in (7) by different estimates of it. Among these, the Gauss-Newton method is preferred for solving nonlinear least squares problems [8]. Consider a nonlinear least squares problem:

$$
f(x)=\frac{1}{2} \sum_{k} r_{k}(x)^{2} .
$$

The Hessian of $f(x)$ is

$$
\nabla^{2} f(x)=\sum_{k} \nabla r_{k}(x) \nabla r_{k}(x)^{T}+\sum_{k} r_{k}(x) \nabla^{2} r_{k}(x) .
$$

In the Gauss-Newton method, one discards the secondorder term to use the approximate Hessian:

$$
\tilde{\nabla}^{2} f(x)=\sum_{k} \nabla r_{k}(x) \nabla r_{k}(x)^{T}
$$

to replace the Hessian $\nabla^{2} f\left(x_{c}\right)$ in (8) to compute the next iterate $x_{+}$. The Gauss-Newton method has quadratic convergence for zero residual problems because the discarded term $\sum_{k} r_{k}(x) \nabla^{2} r_{k}(x)$ is negligible in those cases. However, the Gauss-Newton method only converges linearly or may not converge at all for large residual problems because the Hessian is poorly approximated in these situations [8].

\subsection{Surface Fitting as a Separable Problem}

The surface fitting problem can be formulated as a separable nonlinear least squares problem with the following objective function:

$$
\begin{aligned}
F(\mathcal{P}, \mathcal{U}) & =\sum_{k=1}^{m} F_{k}(\mathcal{P}, \mathcal{U})+F_{s}(\mathcal{P}) \\
& =\frac{1}{2} \sum_{k=1}^{m}\left\|S_{k}(\mathcal{P})-V\left(u_{k}, v_{k}\right)\right\|^{2}+F_{s}(\mathcal{P})
\end{aligned}
$$

where $S_{k}$ is a linear function of the control points $\mathcal{P}$. To simplify the notation, we denote $S=S_{k}(\mathcal{P}),(u, v)=\left(u_{k}, v_{k}\right)$, and $E=S-V(u, v)$. Then, $F_{k}=\frac{1}{2} E^{T} E$. In the following, we will use $\mathcal{P}, u$, and $v$ as subscripts to denote derivatives with respect to these variables.

Since $V(u, v)$ is the foot point of the sample point $S$ to the target surface $\Gamma_{T}, E$ is perpendicular to the tangent plane of $\Gamma_{T}$ at $V(u, v)$. It follows that

$$
\begin{aligned}
& (S-V(u, v))^{T} V_{u}=E^{T} V_{u}=0, \\
& (S-V(u, v))^{T} V_{v}=E^{T} V_{v}=0 .
\end{aligned}
$$

These two equations are constraints tying the variables $(u, v)$ to the control points $\mathcal{P}$. In fact, the two equations in (13) are also necessary conditions for the objective function $F$ to have a local minimum, since the partial derivatives of $F_{k}$ with respect to $u$ and $v$ are

$$
\begin{aligned}
\partial F_{k} / \partial u & =(S-V(u, v))^{T} E_{u}, \\
\partial F_{k} / \partial v & =(S-V(u, v))^{T} E_{v},
\end{aligned}
$$

and noting that $E_{u}=-V_{u}$ and $E_{v}=-V_{v}$. Therefore, the introduction of these constraints does not preclude any minimizer of the original optimization problem.

We first discuss the convergence behavior of PDM. PDM performs the following two alternating steps iteratively: 1) solving a linear system of equations to obtain the variable control points $P_{i}$ while fixing the parameter values $\left(u_{k}, v_{k}\right)$ and 2) computing foot points to find the $\left(u_{k}, v_{k}\right)$ with the fixed control points $P_{i}$. The first step decreases the value of the objective function but moves away from the constraints (13). The second step moves the iterate back to give a feasible point satisfying the constraints (13). This is, in fact, the alternating method for solving a separable and constrained nonlinear problem and is known to have linear convergence [7].

It can be shown that TDM uses a Gauss-Newton step, and SDM is equivalent to the Newton method. The proofs of these facts are given in the appendices in order to have a better flow of discussion.

\subsection{Regularization and Step-Size Control}

The Gauss-Newton method works poorly for large residual problems, so the LM method, which is a regularized version of the Gauss-Newton method, is normally used [8]. The essence of the LM method is that the local quadratic model is trusted only within a small enough neighborhood of the current point $x_{c}$, defined by the constraint $\|s\| \leq \triangle_{k}$, where $s$ is the step. The selection of the value of $\triangle_{k}$ depends on the degree of the agreement between the local model and the objective function. The optimality condition for this constrained optimization gives

$$
\left(\nabla^{2} f\left(x_{c}\right)+\nu_{c} I\right) s=-\nabla f\left(x_{c}\right) .
$$

The Hessian is then approximated as

$$
\nabla^{2} f(x) \approx \sum_{k} \nabla r_{k}(x) \nabla r_{k}(x)^{T}+\nu_{c} I
$$

In other words, $s$ is computed by replacing $\nabla^{2} f\left(x_{c}\right)$ in (8) by $\sum_{k} \nabla r_{k}(x) \nabla r_{k}(x)^{T}+\nu_{c} I$.

In LM regularization, the gain ratio is monitored, which is the ratio of the actual decrease in the objective function to the decrease predicted by the local model [36]. If the gain 


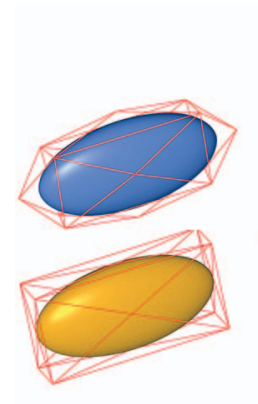

(a)

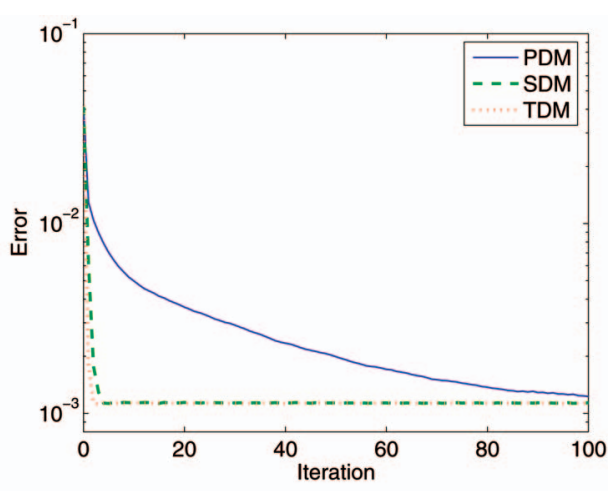

(b)

Fig. 4. (Example 1). (a) Top: Target shape and initial mesh. Bottom: Optimized mesh and surface by SDM. (b) Error curves.

ratio is small, meaning that the current model is a poor approximation to the goal function, $\nu_{c}$ is increased so that the next step will be closer to the steepest descent direction, and the step size is reduced. If the gain ratio is large, meaning that the current model is a good approximation to the goal function, $\nu_{c}$ is decreased so that the next step will be closer to a Gauss-Newton step. By monitoring the agreement between the local model and the actual objective function, this approach tries to get the advantages of both the steepest descent method and the Gauss-Newton method. For all values of $\nu_{c}$, called the LM parameter, the coefficient matrix is positive definite. Note that both the direction and the step size are modified in the LM method. In the same vein, we will also consider the LM regularization of SDM in the next section.

Besides the LM method, the Armijo method [8] is also commonly used for guaranteeing convergence. After the direction of a step $s$ has been determined by a particular method (PDM, SDM, or TDM), the step size is decided by performing a line search. Although the Armijo method improves convergence, extra goal function and gradient evaluations are required, and these increase the computational time. Different from the LM method, only the step size is modified in the Armijo method. The effectiveness of the LM method and the Armijo method will be investigated in the experiments in Section 4.

\section{EXPERIMENTS}

We will present test examples computed by PDM, TDM, and SDM to observe and confirm the convergence behavior of the three methods, as influenced by the initial control mesh specification, smoothness term, and step control methods (that is, the LM method and the Armijo rule.) We will also present examples of subdivision surface reconstruction from complex target shapes. All experiments were run on a PC with an Intel Xeon 2.8-GHz CPU and a 2.00-Gbyte RAM. All data sets are first scaled uniformly to fit into a rectangular box with the longest side equals to 1.0.

\subsection{Initial Mesh and Sharp Feature}

We first consider applying PDM, TDM, and SDM to a data set with two different initial control meshes, without the smoothness term or regularization. Here, no new control points are added during optimization.

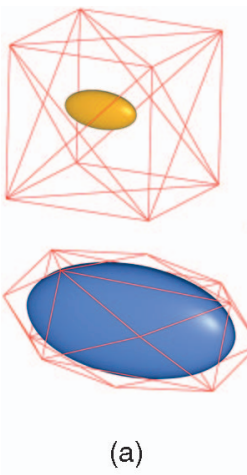

(a)

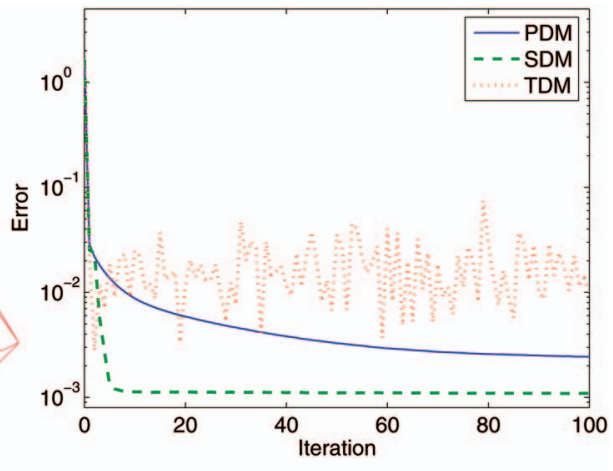

(b)
Fig. 5. (Example 2). (a) Top: Target shape and initial mesh. Bottom: Optimized mesh and surface by SDM. (b) Error curves.

Example 1 (Refer to Fig. 4). The target shape is an ellipsoid with semiprincipal axes being $0.25,0.5$, and 1.0 , and the initial mesh has 14 control points, as shown in Fig. 4 . The optimized surface by SDM is shown in Fig. 4a; the optimized surface by TDM is similar to that by SDM and is therefore not shown. The error curves are shown in Fig. 4b.

Example 2 (Refer to Fig. 5). The same target shape is used here as in Example 1, but the initial control mesh is now farther away from the target shape, as shown in Fig. 5a. The surface reconstructed with SDM is also shown. The error curves of PDM, TDM, and SDM are shown in Fig. 5b. TDM does not converge for this data set.

Discussion. Examples 1 and 2 show that SDM converges much faster than PDM. TDM has a similar convergence rate as SDM but may easily become unstable if the initial mesh is far away from the target shape. That is because TDM, using a Gauss-Newton step, discards from the Hessian the part $r(x) \nabla^{2} r(x)$ that is related to the curvature and residue; here, $r(x)$ reflects the distance between the initial fitting surface and the target shape. Therefore, TDM should always be used with LM regularization for stable convergence, as will be seen shortly.

Example 3 (Refer to Fig. 6). Here, we consider an elongated ellipsoid with two sharp ends, with semiprincipal axes being $0.125,0.25$, and 4.0, as shown in Fig. 6a with the initial mesh. We use this target shape to test PDM, TDM, and SDM in the presence of sharp features. The optimized subdivision surface by SDM is also shown. The optimized surface by TDM is similar to that by SDM and is therefore not shown. The error curves of PDM, TDM, and SDM are shown in Fig. 6b. We see that SDM and TDM have similar convergence behaviors, and PDM converges to a poor local minimum with a larger residual error.

\subsection{Smoothness Term and Multistage Optimization}

In this section, we consider the effects of the smoothness term on convergence behaviors and insertion of new control points.

Example 4 (Refer to Figs. 5a and 7a). The data set and initial control mesh used here are the same as those in Example 2 (see Fig. 5a), where TDM fails to converge. Now, we want to observe whether the introduction of a smoothness term 


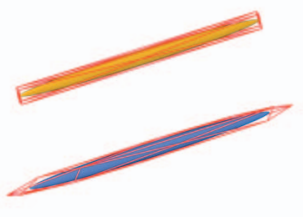

(a)

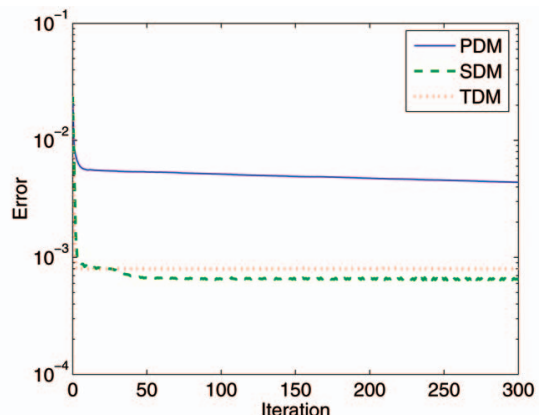

(b)

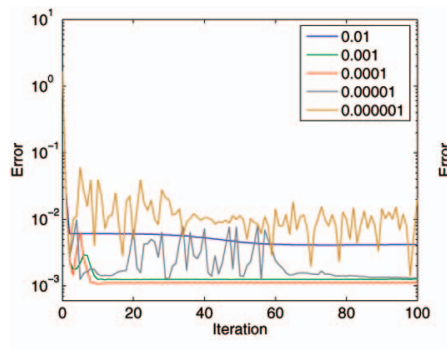

(a)

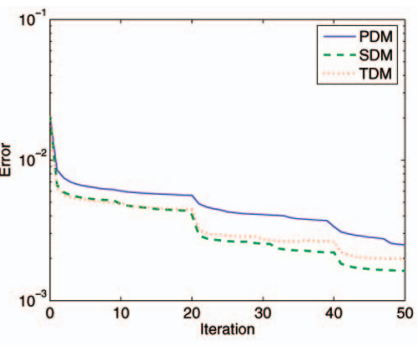

(b)
Fig. 7. (a) Error of TDM for Example 4. (b) Error for Example 5.

\subsection{LM Regularization and the Armijo Rule}

Since TDM uses a Gauss-Newton step, it exhibits instability if used without any regularization. In this section, we apply LM regularization to TDM and also to SDM to observe its effect on the convergence of the two methods.

make the convergence of TDM stable. The smoo term is defined in Section 2.4. We tested different values of coefficients $\lambda=10^{-i}, i=2,3,4,5,6$, resulting in the error curves of TDM shown in Fig. 7a.

Discussion. In this example, $\lambda=0.001$ or 0.0001 leads to stable convergence with a small fitting error. Bigger values of $\lambda$ make the surface too "stiff," giving large fitting errors, whereas smaller values of $\lambda$ fail to make TDM stable. We remark that, except for trial and error or methods based on user assistance, there is currently no commonly accepted general scheme that can automatically determine the coefficient of the smoothness term in the context of curve or surface fitting.

Example 5 (Refer to Fig. $7 \mathrm{~b}$ ). Here, we will test the effects of adaptively adding new control points and reducing the smoothness coefficient progressively at different stages of the fitting process, using PDM, TDM, and SDM. The target shape is the ball joint model containing 137,062 points (Fig. 9), and the initial control mesh has 128 control points. The initial smoothness coefficient is 0.01 . Throughout optimization, new control points are inserted at regions of large errors progressively. The final number of control points at the 50th iteration is 202, 151, and 199 for PDM, TDM, and SDM, respectively. The smoothness coefficient is decreased to 0.001 and 0.0001 at the 20th and 40th iterations, respectively. Fig. $7 \mathrm{~b}$ shows the error curves of PDM, TDM, and SDM. The obvious reduction of the fitting error can be observed due to the control point insertion and the smoothing coefficient adjustment. During each stage between the insertions of control points, SDM and TDM are again more efficient than PDM.
Example 6 (LM regularization of TDM. Refer to Figs. 4a and 8a). Here, the target shape and initial control mesh are the same as in Example 1, as shown in Fig. 4a. No LM regularization. Fig. 8a shows the error curves for TDMLM and TDM. TDMLM takes $0.551 \mathrm{sec}$ to get an $E_{r m s}$ smaller than 0.002. The error curves in Fig. 8a indicate that TDMLM delivers both fast convergence and a monotonic descent of the fitting error, whereas the error of TDM is not monotonically decreasing.

Example 7 (LM regularization of TDM. Refer to Figs. 5a and $\mathbf{8 b})$. Here, the target shape and the initial control mesh are the same as that used in Example 2, as shown in Fig. 5a. Recall that TDM fails to converge in that example. Now, we apply TDMLM without a smoothing term. Fig. $8 \mathrm{~b}$ shows the error curves of TDMLM and TDM. Clearly, TDMLM converges fast and stably. TDMLM takes $1.593 \mathrm{sec}$ to have an $E_{r m s}$ smaller than 0.002 .

Example 8 (LM regularization of SDM. Refer to Figs. 4a and 8c). Here, we apply LM regularization to SDM for the same target shape and initial mesh as in Example 6, as shown in Fig. 4a. The regularized SDM will be denoted by SDMLM. Fig. $8 \mathrm{c}$ shows the error curves of SDM and SDMLM, where the SDM error curve here is the rescaled version of the same SDM error curve in Fig. 4. It can be seen that the SDM error curve is actually not monotonically descending, but that of SDMLM is. SDMLM takes $1.011 \mathrm{sec}$ to get an $E_{r m s}$ smaller than 0.002.

Example 9 (LM regularization of SDM. Refer to Figs. 5a and $\mathbf{8 d ) . ~ I n ~ t h i s ~ e x a m p l e , ~ S D M ~ a n d ~ S D M L M ~ a r e ~ a p p l i e d ~}$ smoothing term is used. Let TDMLM denote TDM with
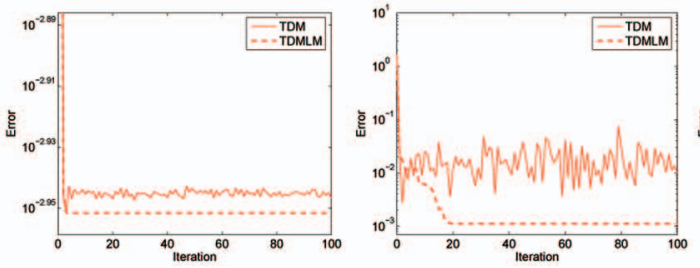

(a)

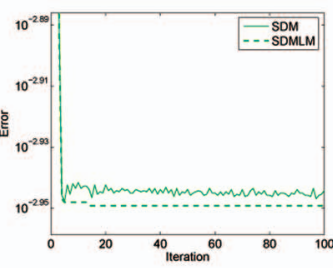

(c)

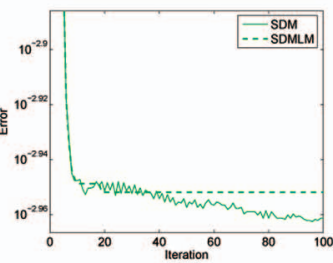

(d)

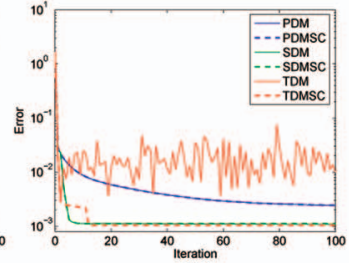

(e)

Fig. 8. Error curves (a) for Example 6 (this is the zoom-in view of the TDM error curve in Fig. 4), (b) for Example 7, (c) for Example 8, (d) for Example 9, and (e) for Example 10. 


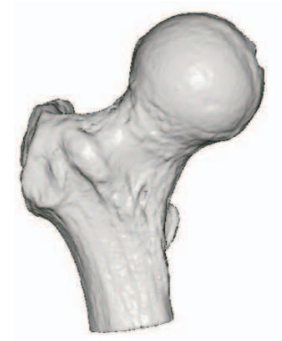

(a)

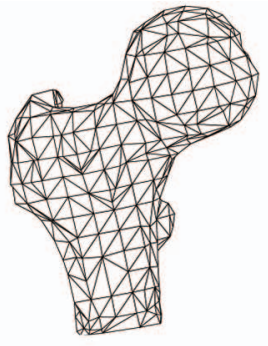

(b)

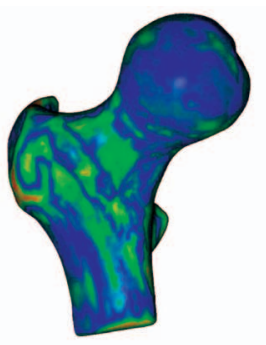

(c)

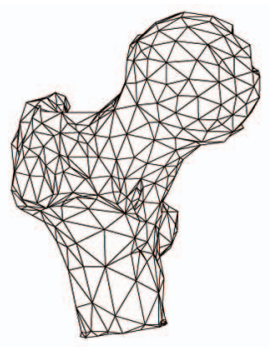

(d)

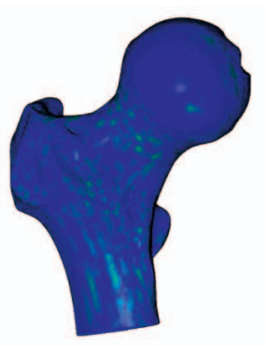

(e)

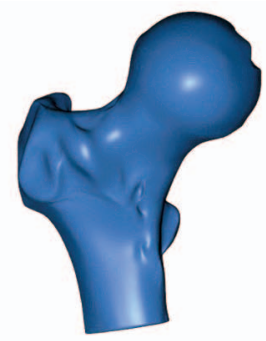

(f)

Fig. 9. Ball Joint: (a) Point cloud (137,062 points; dimension: $0.87 \times 0.50 \times 1)$. (b) Initial mesh (416 control points). (c) Initial surface. (d) Final mesh (551 control points). (e) Final surface. (f) Shaded subdivision surface. Maximum error: 0.0064; RMS error: 0.0009.

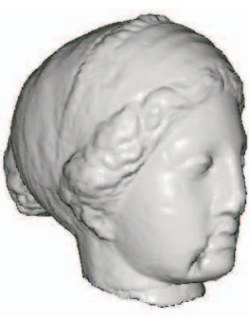

(a)

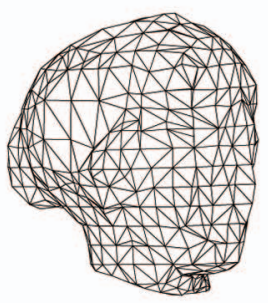

(b)

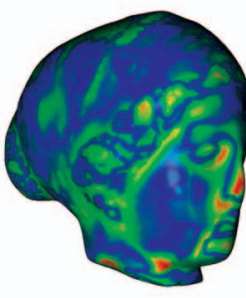

(c)

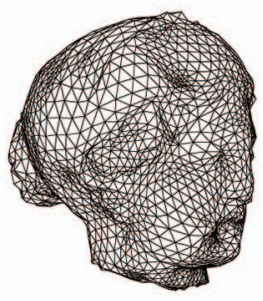

(d)

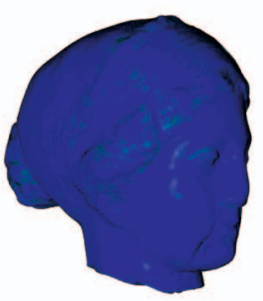

(e)

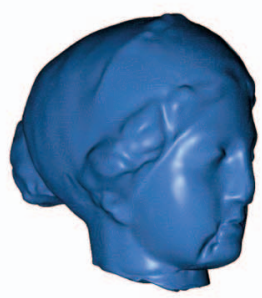

(f)

Fig. 10. Igea: (a) Point cloud (134,345 points; dimension: $0.70 \times 1 \times 1)$. (b) Initial mesh $(526$ control points). (c) Initial surface. (d) Final mesh (2,464 control points). (e) Final surface. (f) Shaded subdivision surface. Maximum error: 0.0036; RMS error: 0.0005.

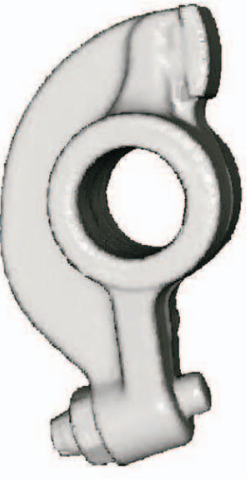

(a)

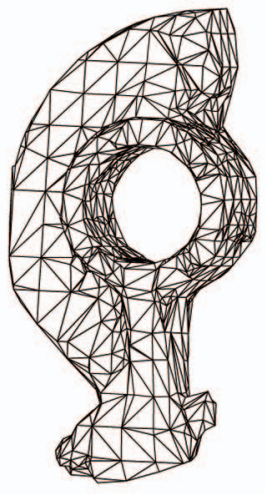

(b)

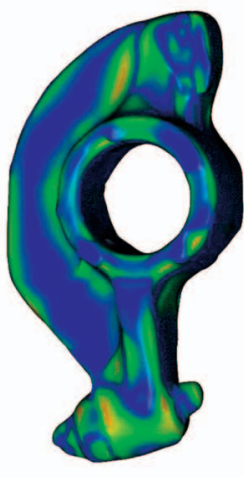

(c)

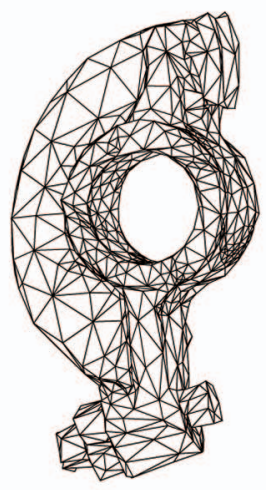

(d)

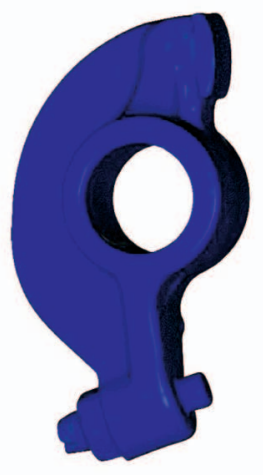

(e)

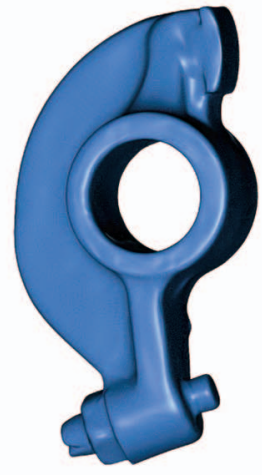

(f)

Fig. 11. RockerArm: (a) Point cloud (40,177 points; dimension: $0.51 \times 1 \times 0.30)$. (b) Initial mesh (870 control points). (c) Initial surface. (d) Final mesh (950 control points). (e) Final surface. (f) Optimized subdivision surface. Maximum error: 0.0029; RMS error: 0.0003.

to the same target shape and initial control mesh as in Example 7, as shown in Fig. 5a. The error curves of SDM and SDMLM are shown in Fig. 8d. Again, we see that SDMLM leads to more stable convergence than SDM without regularization.

Example 10 (Refer to Figs. 5 and 8e). In this example, stepsize control using the Armijo rule is applied to every step of PDM, TDM, and SDM. The target shape and the initial mesh are the same as in Example 2 (shown in Fig. 5). The corresponding variants of PDM, TDM, and SDM with step-size control are called PDMSC, TDMSC, and SDMSC, respectively. Fig. 8e shows the error curves of PDM, TDM, SDM, and their variants. We see that the stability of TDM is improved greatly by step-size control, whereas PDMSC and SDMSC have similar convergence behaviors to PDM and SDM. PDMSC has an $E_{r m s}$ larger than 0.002 after 100 iterations (4.138 sec), whereas SDMSC and TDMSC just take five iterations (0.240 sec) and 12 iterations $(0.812 \mathrm{sec})$ to obtain an $E_{r m s}$ smaller than 0.002 , respectively.

Discussion. From the three preceding examples, we conclude that LM regularization is helpful to the stabilization of TDM and SDM, and it ensures a monotonic decrease of fitting errors. Step-size control by the Armijo rule is also very effective for TDM, but much less effective for PDM and SDM, which are often already quite stable even without step-size control, though step-size control does ensure a monotonic decrease of SDM.

\subsection{Surfaces from Complex Target Shapes}

In this section, we will show that our fitting method works effectively as well for complex target shapes. Figs. 9, 10, 11, 12 , and 13 show the data sets for a ball joint, a head (Igea), a rocker arm, a bunny, and a buddha (data sources: http:/ / www.cyberware.com (Igea, the ball joint, and the rocker arm) and http://www-graphics.stanford.edu/data/3Dscanrep/ 


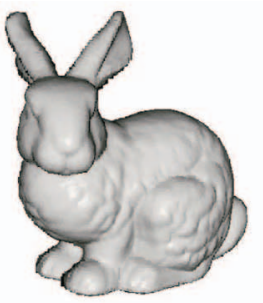

(a)

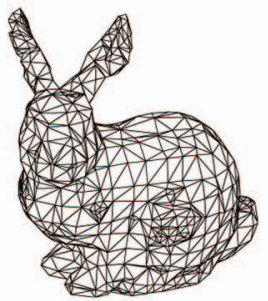

(b)

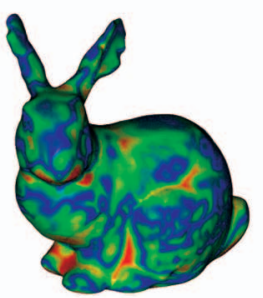

(c)

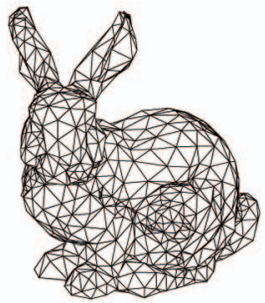

(d)

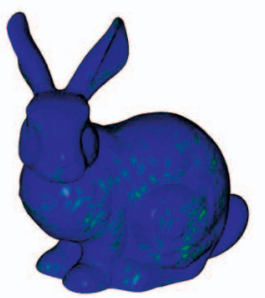

(e)

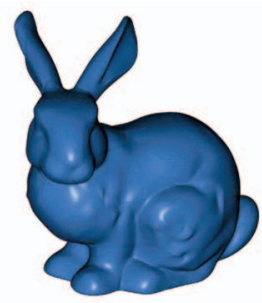

(f)

Fig. 12. Bunny: (a) Point cloud (35,201 points; dimension: $1 \times 0.78 \times 0.99)$. (b) Initial mesh (919 control points). (c) Initial surface. (d) Final mesh (996 control points). (e) Final surface. (f) Optimized subdivision surface. Maximum error: 0.0082; RMS error: 0.0009.

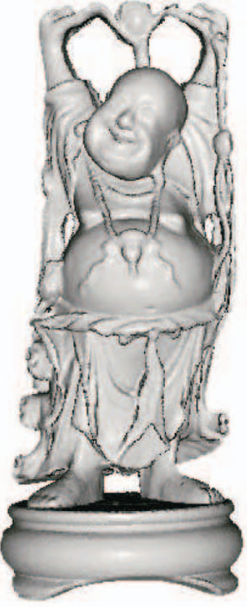

(a)

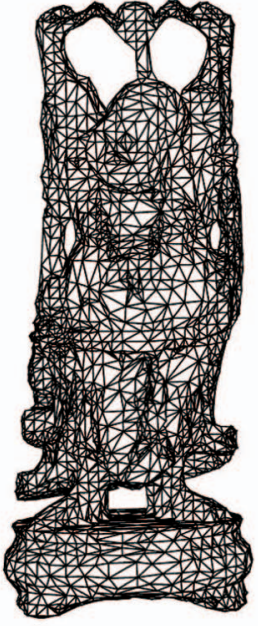

(b)

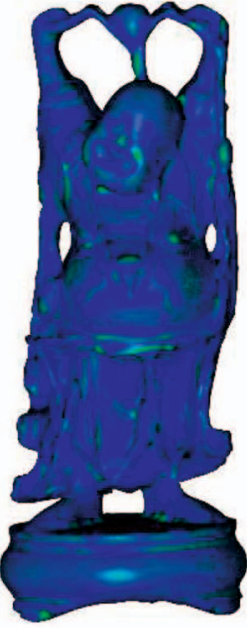

(c)

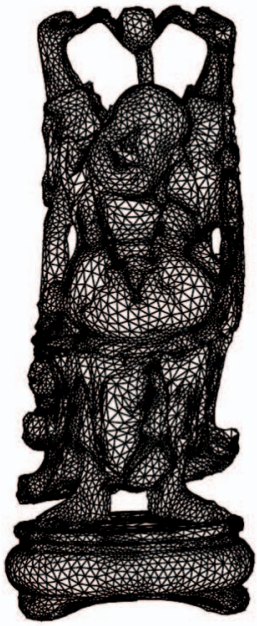

(d)

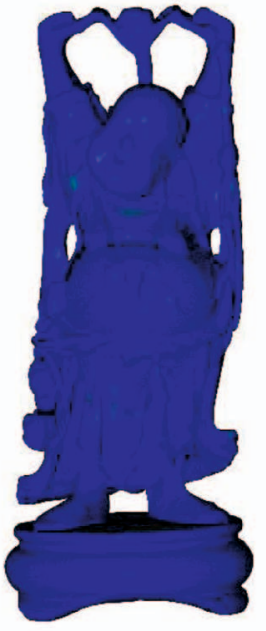

(e)

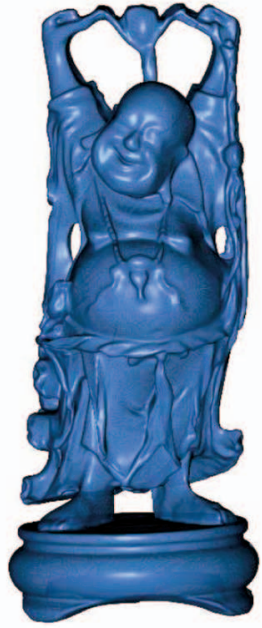

(f)

Fig. 13. Buddha: (a) Point cloud (543,652 points; dimension: $0.41 \times 0.41 \times 1)$. (b) Initial mesh $(4,662$ control points). (c) Initial surface. (d) Final mesh $(18,715$ control points). (e) Final surface. (f) Optimized subdivision surface. Maximum error: 0.0043; RMS error: 0.0003.

(the bunny and the buddha)). The figures show the initial meshes, the optimized control meshes by SDM, the initial and optimized subdivision surfaces with color error coding, and the shaded optimized subdivision surfaces. The color code of a data point is interpolated in a piecewise linear manner from blue, green, yellow, and red corresponding to local error values (see Section 2.2) 0.0, 0.0066666, 0.0133333, and 0.02 , respectively.

Table 1 gives the timing data for the preprocessing steps. Neighboring points within a distance of 0.03 from a data point $V_{k}$ are used for computing the curvatures at $V_{k}$. Table 4 shows the error statistics. The numbers in the \# of contr. pts field refer to the number of control points in the initial control meshes and the final optimized control meshes. The numbers in the smooth. coeff. field refer to the initial and the final values for the smoothing term coefficient. Table 2 shows the breakdown of the time used in different tasks in the optimization. The total time does not include the time on precomputation. All the five examples were computed with SDM.

In Table 2, we observe that the time for generating entries of the matrix of the linear equations is substantial when compared with other parts. Note that the number of data points affects mainly the time used for the preprocessing steps but does not affect much the time used in the optimization step, which is mainly determined by the number of control points.

Finally, we would like to compare the fitting result of the Igea model in Fig. 10 with that produced in [24]. Table 3 shows that SDM obtains comparably small $E_{m}$ and $E_{r m s}$ as by the method in [24], using a significantly shorter period of time. (The PC on which we ran this experiment has the same specifications as the one used in [24].) However, we note that the projection direction for fitting error evaluation is different from that in our
TABLE 1

Time Statistics (in Seconds) for Precomputation

\begin{tabular}{|c|c|c|c|}
\hline & $\begin{array}{c}\text { \# of data } \\
\text { points }\end{array}$ & Curvatures & $\begin{array}{c}\text { Distance } \\
\text { fields }\end{array}$ \\
\hline Ball joint & 137,062 & $95.83 \mathrm{~s}$ & $52.51 \mathrm{~s}$ \\
Igea & 134,345 & $36.07 \mathrm{~s}$ & $215.69 \mathrm{~s}$ \\
Rocker arm & 40,177 & $14.91 \mathrm{~s}$ & $70.48 \mathrm{~s}$ \\
Bunny & 35,201 & $212.74 \mathrm{~s}$ & $148.26 \mathrm{~s}$ \\
Buddha & 543,652 & $4837.67 \mathrm{~s}$ & $200.66 \mathrm{~s}$ \\
\hline
\end{tabular}

TABLE 2

Time Breakdown (in Seconds)

\begin{tabular}{|c|c|c|c|c|}
\hline & $\begin{array}{c}\text { Eqn. } \\
\text { setup }\end{array}$ & $\begin{array}{c}\text { Eqn. } \\
\text { solving }\end{array}$ & $\begin{array}{c}\text { Err. } \\
\text { evaluation }\end{array}$ & $\begin{array}{c}\text { Ttl. } \\
\text { time }\end{array}$ \\
\hline Ball joint & 38 & 3 & 31 & 73 \\
Igea & 83 & 5 & 63 & 152 \\
Rocker arm & 41 & 6 & 30 & 78 \\
Bunny & 39 & 5 & 32 & 77 \\
Buddha & 1,134 & 32 & 641 & 1,808 \\
\hline
\end{tabular}


TABLE 3

Comparison with the Approach in [24] for the Igea Model

\begin{tabular}{|c|c|c|c|c|}
\hline & $\begin{array}{c}\text { Final \# of } \\
\text { control points }\end{array}$ & $\begin{array}{c}E_{m} \\
(\%)\end{array}$ & $\begin{array}{c}E_{r m s} \\
(\%)\end{array}$ & $\begin{array}{c}\text { Time taken } \\
\text { (min:sec) }\end{array}$ \\
\hline Result in [24] & 1,553 & 0.247 & 0.05755 & $8: 29$ \\
Our result & 2,385 & 0.229 & 0.03472 & $2: 32$ \\
\hline
\end{tabular}

The errors $E_{m}$ and $E_{r m s}$ are expressed in percentage of the diagonal of the model.

TABLE 4

Optimization Setup

\begin{tabular}{|c|c|c|c|c|c}
\hline & $\begin{array}{c}\# \text { of } \\
\text { iter. }\end{array}$ & $\begin{array}{c}\# \text { of } \\
\text { contr. pts. }\end{array}$ & $\begin{array}{c}\text { smooth. } \\
\text { coeff. }\end{array}$ & $E_{m}$ & $E_{r m s}$ \\
\hline Ball joint & 29 & 416,551 & $10^{-2}, 10^{-4}$ & 0.0064 & 0.0009 \\
Igea & 14 & $526,2,385$ & $10^{-2}, 10^{-4}$ & 0.0036 & 0.0005 \\
Rocker arm & 15 & 870,950 & $10^{-2}, 10^{-5}$ & 0.0029 & 0.0003 \\
Bunny & 14 & 919,996 & $10^{-2}, 10^{-5}$ & 0.0082 & 0.0009 \\
Buddha & 9 & $4,662,18,715$ & $10^{-3}, 10^{-5}$ & 0.0043 & 0.0003 \\
\hline
\end{tabular}

approach. In [24], target data points are projected onto the fitting subdivision surface. In our approach, sample points on the subdivision surface are projected onto the target shape, following the framework in [6].

\section{Discussion AND Conclusion}

We have presented a comprehensive study on a class of three methods for fitting subdivision surfaces to $3 \mathrm{D}$ data points, both theoretically and experimentally, from the optimization point of view. There are a variety of other optimization techniques that can be applied to shape fitting and further efforts should be made on understanding these methods as well.

There are currently two different variants of SDM, along with TDM and PDM. The study presented in this paper is based on the original framework proposed by Pottmann and Leopoldseder [6], and the other variant has been recently proposed by Wang et al. [28] in the setting of Bspline curve fitting, where the connections of PDM, TDM, and SDM to the standard optimization techniques are also studied. Although these two different frameworks have apparently been motivated by the same geometric intuition, they need to be distinguished carefully, not merely because of the difference in the dimensions of their working spaces. In the framework used in [28], the data points are projected onto the fitting curve for error measurement, whereas in the present paper, sampled points on the fitting surface are projected onto a fixed target shape defined by data points for error measurement. This difference means that the analysis and results in [28] do not apply to the setting in this paper. For example, here, we have shown that the SDM method follows directly from the Newton method, whereas the Hessian has to be greatly simplified in order to derive the SDM method in [28]. In addition, the present paper is featured by extensive experimental study of effects of many practical aspects of optimization, including control point insertion, smoothness terms, LM regularization, and the Armijo rule for step-size control.

In the three optimization methods considered here, since sample points on the fitting surface are projected onto the target shape for setting up error functions, they essentially assume that the target shape is not very noisy or sparse so that the estimated normal and curvatures are reasonably accurate. The advantage of this treatment is that foot points, normal, and curvature information can be computed efficiently with the aid of preprocessing the fixed target shape. However, the drawback is the limit on the application of these methods to fitting a surface to noisy and sparse data points. One way of addressing this limitation is to use instead the methods (PDM, TDM, and SDM) presented in [24], [28]. Note that in that case, the computation of foot points, normal, and curvature information has to be performed on an iteratively updating fitting subdivision surface and will therefore be relatively time consuming.

\section{APPENDIX A}

\section{TDM AND THE Gauss-NeWTON Method}

We shall show that TDM uses a Gauss-Newton iteration. We first compute the gradient of $F$ by taking into account the dependence of the foot point parameters $(u, v)$ on the control points $\mathcal{P}$ through the constraints (13). Since $F_{k}=\frac{1}{2} E^{T} E$, due to the constraints (13), we obtain the gradient vector

$$
\nabla_{\mathcal{P}} F_{k}=\left(S_{P}^{T}-\nabla_{\mathcal{P}} u V_{u}^{T}-\nabla_{\mathcal{P}} v V_{v}^{T}\right) E=S_{\mathcal{P}}^{T} E .
$$

Here, $\nabla_{\mathcal{P}} F_{k}$ is a $3 n$ vector, where $n$ is the number of control points, and $S_{\mathcal{P}}^{T}$ is a $3 n$ by 3 matrix. Let $F_{k}(x)=\frac{1}{2} f_{k}^{2}=\frac{1}{2} E^{T} E$, where $f_{k}=\|E\|$. Since $\nabla_{\mathcal{P}} F_{k}=f_{k} \nabla_{\mathcal{P}} f_{k}$, we have, by (16)

$$
\nabla_{\mathcal{P}} f_{k}=\frac{\nabla_{\mathcal{P}} F_{k}}{f_{k}}=S_{P}^{T} \frac{E}{f_{k}}=S_{P}^{T} \frac{E}{\|E\|}=S_{P}^{T} N,
$$

where $N$ is the unit normal vector of the target surface $\Gamma_{T}$ at the foot point $V$.

By (17), we have $\nabla_{\mathcal{P}} f_{k}\left(\nabla_{\mathcal{P}} f_{k}\right)^{T}=S_{\mathcal{P}}^{T} N N^{T} S_{\mathcal{P}}$. In the second-order Taylor expansion of $F_{k}$ at $\mathcal{P}_{c}$, replacing the Hessian by $\nabla_{\mathcal{P}} f_{k}\left(\nabla_{\mathcal{P}} f_{k}\right)^{T}$ yields

$$
\begin{aligned}
& F_{k}\left(\mathcal{P}_{c}\right)+\nabla_{\mathcal{P}} F_{k}\left(\mathcal{P}_{c}\right)^{T} \Delta \mathcal{P}+\frac{1}{2} \Delta \mathcal{P}^{T} \nabla_{\mathcal{P}}^{2} F_{k}\left(\mathcal{P}_{c}\right) \Delta \mathcal{P} \\
\approx & F_{k}\left(P_{c}\right)+\nabla_{\mathcal{P}} F_{k}\left(\mathcal{P}_{c}\right)^{T} \Delta \mathcal{P}+\frac{1}{2} \Delta \mathcal{P}^{T} S_{\mathcal{P}}^{T} N N^{T} S_{\mathcal{P}} \Delta \mathcal{P} \\
= & \frac{1}{2}\left(S_{c}-V_{c}\right)^{T}\left(S_{c}-V_{c}\right)+\left(S_{c}-V_{c}\right)^{T} S_{\mathcal{P}} \Delta \mathcal{P} \\
+ & \frac{1}{2} \Delta \mathcal{P}^{T} S_{\mathcal{P}}^{T} N N^{T} S_{\mathcal{P}} \Delta \mathcal{P} \\
= & \frac{1}{2}\left(S_{c}-V_{c}\right)^{T} N N^{T}\left(S_{c}-V_{c}\right) \\
+ & \left(S_{c}-V_{c}\right)^{T} N N^{T} S_{\mathcal{P}} \Delta \mathcal{P}+\frac{1}{2} \Delta \mathcal{P}^{T} S_{\mathcal{P}}^{T} N N^{T} S_{\mathcal{P}} \Delta \mathcal{P} \\
= & \frac{1}{2}\left(S_{c}+S_{\mathcal{P}} \Delta \mathcal{P}-V_{c}\right)^{T} N N^{T}\left(S_{c}+S_{\mathcal{P}} \Delta \mathcal{P}-V_{c}\right) \\
= & \frac{1}{2}\left(S-V_{c}\right)^{T} N N^{T}\left(S-V_{c}\right)=\frac{1}{2}\left[\left(S-V_{c}\right)^{T} N\right]^{2} \\
= & \frac{1}{2} \times \text { TD error term, }
\end{aligned}
$$

where the last equality follows from (4). Since $\left(S_{c}-V_{c}\right)$ and $N$ above are parallel, we have made use of the fact that

$$
\left(S_{c}-V_{c}\right)^{T}\left(S_{c}-V_{c}\right)=\left(S_{c}-V_{c}\right)^{T} N N^{T}\left(S_{c}-V_{c}\right)
$$

and

$$
\begin{aligned}
& \left(S_{c}-V_{c}\right)^{T} S_{\mathcal{P}} \Delta \mathcal{P}=f_{k} N^{T} S_{\mathcal{P}} \Delta \mathcal{P} \\
& =f_{k} N^{T} N N^{T} S_{\mathcal{P}} \Delta \mathcal{P}=\left(S_{c}-V_{c}\right)^{T} N N^{T} S_{\mathcal{P}} \Delta \mathcal{P} .
\end{aligned}
$$

Hence, by (18), TDM uses a Gauss-Newton step. 


\section{APPENDIX B}

\section{SDM AND THE NewTON MethOd}

Now, we are going to show that the SD error term is given by the Newton iteration, after appropriate modification to make the Hessian positive semidefinite. Differentiating $\nabla_{\mathcal{P}} F_{k}$ in (16) yields the Hessian as

$$
\begin{aligned}
\nabla_{\mathcal{P}}^{2} F_{k} & =\left(S_{\mathcal{P}}^{T}-\nabla_{\mathcal{P}} u V_{u}^{T}-\nabla_{\mathcal{P}} v V_{v}^{T}\right) S_{\mathcal{P}}+E^{T} S_{\mathcal{P}} \\
& =\left(S_{\mathcal{P}}^{T}-\nabla_{\mathcal{P}} u V_{u}^{T}-\nabla_{\mathcal{P}} v V_{v}^{T}\right) S_{\mathcal{P}} .
\end{aligned}
$$

Here, $E^{T} S_{\mathcal{P P}}=0$, since the sample point $S$ is a linear combination of control points $\mathcal{P}$.

Without loss of generality, suppose that $V(u, v)$ is a local regular parameterization of the target surface $\Gamma_{T}$ such that $V_{u}=T_{1}$ and $V_{v}=T_{2}$, which are the unit principal direction vectors of $\Gamma_{T}$ at the current foot point $V_{c}$, and then, there is $V_{u}^{T} V_{v}=0$. Differentiating the constraints (13) with respect to $\mathcal{P}$ yields

$$
\begin{aligned}
& 0=\left(\nabla_{\mathcal{P}} u V_{u u}^{T}+\nabla_{\mathcal{P}} v V_{v u}^{T}\right) E \\
& +\left(S_{\mathcal{P}}^{T}-\nabla_{\mathcal{P}} u V_{u}^{T}-\nabla_{\mathcal{P}} v V_{v}^{T}\right) V_{u} \\
& =\left(V_{u u}^{T} E-V_{u}^{T} V_{u}\right) \nabla_{\mathcal{P}} u+S_{\mathcal{P}}^{T} V_{u}+V_{v u}^{T} E \nabla_{\mathcal{P} v}
\end{aligned}
$$

and

$$
\begin{aligned}
& 0=\left(\nabla_{\mathcal{P}} v V_{v v}^{T}+\nabla_{\mathcal{P}} u V_{u v}^{T}\right) E \\
& +\left(S_{\mathcal{P}}^{T}-\nabla_{\mathcal{P}} u V_{u}^{T}-\nabla_{\mathcal{P}} v V_{v}^{T}\right) V_{v} \\
& =\left(V_{v v}^{T} E-V_{v}^{T} V_{v}\right) \nabla_{\mathcal{P}} v+S_{\mathcal{P}}^{T} V_{v}+V_{u v}^{T} E \nabla_{\mathcal{P}} u .
\end{aligned}
$$

From (20) and (21), we obtain

$$
\nabla_{\mathcal{P}} u=-\frac{S_{\mathcal{P}}^{T} V_{u}+V_{v u}^{T} E \nabla_{\mathcal{P}} v}{V_{u u}^{T} E-V_{u} T V_{u}}
$$

and

$$
\nabla_{\mathcal{P}} v=-\frac{S_{\mathcal{P}}^{T} V_{v}+V_{u v}^{T} E \nabla_{\mathcal{P}} u}{V_{v v}^{T} E-V_{v}^{T} V_{v}}
$$

By assumption and differential geometry of surfaces, we have $V_{u}=T_{1}, V_{v}=T_{2}, V_{u u}=\kappa_{1} N, V_{v v}=\kappa_{2} N$, and $E=d N$, where $\kappa_{1}, \kappa_{2}$ are the signed principal curvatures at $V$. With the substitutions in (22) and (23), we obtain $V_{u u}^{T} E-V_{u}^{T} V_{u}=$ $d \kappa_{1}-1$ and $V_{v v}^{T} E-V_{v}^{T} V_{v}=d \kappa_{2}-1$. Furthermore, since $V_{u}^{T} E=0$, differentiating with respect to $v$ yields

$$
V_{u v}^{T} E+V_{u}^{T} E_{v}=V_{u v}^{T} E-V_{u}^{T} V_{v}=0 .
$$

Therefore, $V_{u v}^{T} E=V_{u}^{T} V_{v}=0$. Putting all these together, it follows from (22) and (23) that

$$
\nabla_{\mathcal{P}} u=-\frac{S_{\mathcal{P}}^{T} T_{1}}{d \kappa_{1}-1}, \quad \nabla_{\mathcal{P}} v=-\frac{S_{\mathcal{P}}^{T} T_{2}}{d \kappa_{2}-1} .
$$

Recall the notation $\rho_{1}=\frac{1}{\kappa_{1}}$ and $\rho_{2}=\frac{1}{\kappa_{2}}$ from Section 2.4. Substituting (24) in (19) yields

$$
\begin{aligned}
\nabla_{\mathcal{P}}^{2} F_{k}= & S_{\mathcal{P}}^{T} S_{\mathcal{P}}+\frac{S_{\mathcal{P}}^{T} T_{1} T_{1}^{T} S_{\mathcal{P}}}{d \kappa_{1}-1}+\frac{S_{\mathcal{P}}^{T} T_{2} T_{2}^{T} S_{\mathcal{P}}}{d \kappa_{2}-1} \\
= & S_{\mathcal{P}}^{T}\left(I-T_{1} T_{1}^{T}-T_{2} T_{2}^{T}\right) S_{\mathcal{P}} \\
& +d \kappa_{1} \frac{S_{\mathcal{P}}^{T} T_{1} T_{1}^{T} S_{\mathcal{P}}}{d \kappa_{1}-1}+d \kappa_{1} \frac{S_{\mathcal{P}}^{T} T_{2} T_{2}^{T} S_{\mathcal{P}}}{d \kappa_{2}-1} \\
= & S_{\mathcal{P}}^{T} N N^{T} S_{\mathcal{P}}+\alpha_{1} S_{\mathcal{P}}^{T} T_{1} T_{1}^{T} S_{\mathcal{P}}+\alpha_{2} S_{\mathcal{P}}^{T} T_{2} T_{2}^{T} S_{\mathcal{P}},
\end{aligned}
$$

where $\alpha_{1}=d /\left(d-\rho_{1}\right)$ and $\alpha_{2}=d /\left(d-\rho_{2}\right)$ as in Section 2.4. Here, note that $T_{1} T_{1}^{T}+T_{2} T_{2}^{T}+N N^{T}=I$, and $d, \rho_{1}, \rho_{2}$ are signed values (see Section 2.4.3).

Thus, we have obtained the Hessian $\nabla^{2} F_{k}$. Substituting $\nabla^{2} F_{k}$ in the following second-order Taylor approximation of the objective function, noting that $\left(S_{c}-V_{c}\right)^{T} T_{1}=\left(S_{c}-V_{c}\right)^{T}$ $T_{2}=0$, we obtain

$$
\begin{aligned}
& F_{k}\left(\mathcal{P}_{c}\right)+\nabla_{\mathcal{P}} F_{k}\left(\mathcal{P}_{c}\right)^{T} \Delta \mathcal{P}+\frac{1}{2} \Delta \mathcal{P}^{T} \nabla_{\mathcal{P}}^{2} F_{k}\left(\mathcal{P}_{c}\right) \Delta \mathcal{P} \\
= & \frac{1}{2}\left(S_{c}-V_{c}\right)^{T}\left(S_{c}-V_{c}\right)+\left(S_{c}-V_{c}\right)^{T} S_{\mathcal{P}} \Delta \mathcal{P} \\
& +\frac{1}{2} \Delta \mathcal{P}^{T}\left(S_{\mathcal{P}}^{T} N N^{T} S_{\mathcal{P}}+\alpha_{1} S_{\mathcal{P}}^{T} T_{1} T_{1}^{T} S_{\mathcal{P}}\right. \\
& \left.+\alpha_{2} S_{\mathcal{P}}^{T} T_{2} T_{2}^{T} S_{\mathcal{P}}\right) \Delta \mathcal{P} \\
= & \frac{1}{2}\left(S_{c}-V_{c}\right)^{T} N N^{T}\left(S_{c}-V_{c}\right) \\
& +\left(S_{c}-V_{c}\right)^{T} N N^{T} S_{\mathcal{P}} \Delta \mathcal{P}+\frac{1}{2} \Delta \mathcal{P}^{T} S_{\mathcal{P}}^{T} N N^{T} S_{\mathcal{P}} \Delta \mathcal{P} \\
& +\frac{1}{2} \alpha_{1}\left(S_{c}+S_{\mathcal{P}} \Delta \mathcal{P}-V_{c}\right)^{T} T_{1} T_{1}^{T}\left(S_{c}+S_{\mathcal{P}} \Delta \mathcal{P}-V_{c}\right) \\
& +\frac{1}{2} \alpha_{2}\left(S_{c}+S_{\mathcal{P}} \Delta \mathcal{P}-V_{c}\right)^{T} T_{2} T_{2}^{T}\left(S_{c}+S_{\mathcal{P}} \Delta \mathcal{P}-V_{c}\right) \\
= & \frac{1}{2}\left(S_{c}+S_{\mathcal{P}} \Delta \mathcal{P}-V_{c}\right)^{T} N N^{T}\left(S_{c}+S_{\mathcal{P}} \Delta \mathcal{P}-V_{c}\right) \\
& +\frac{1}{2} \alpha_{1}\left(S-V_{c}\right)^{T} T_{1} T_{1}^{T}\left(S-V_{c}\right) \\
& +\frac{1}{2} \alpha_{2}\left(S-V_{c}\right)^{T} T_{2} T_{2}^{T}\left(S-V_{c}\right) \\
= & \frac{1}{2}\left[\left(S-V_{c}\right)^{T} N\right]^{2}+\frac{1}{2} \alpha_{1}\left[\left(S-V_{c}\right)^{T} T_{1}\right]^{2} \\
& +\frac{1}{2} \alpha_{2}\left[\left(S-V_{c}\right)^{T} T_{2}\right]^{2} \\
= & \frac{1}{2} \hat{f}_{S D, k}^{2},
\end{aligned}
$$

which, up to a constant multiple, is the second-order approximation of the SD given in (5). Thus, the SD error term defined in (6) is derived from the Newton iteration after removing the negative eigenvalues from the full Hessian by setting the negative coefficients in (5), if any, to zero.

When we compute the gradient and Hessian of the global function with respect to $\mathcal{P}$, we treat $\mathcal{P}$ as basic variables and $\mathcal{U}$ as dependent variables. Furthermore, the dependence between $\mathcal{U}$ and $\mathcal{P}$ is expressed by the way we compute the gradient in (16) and the Hessian using the local linear relations (22) and (23). In fact, this local linear dependence between $\mathcal{U}$ and $\mathcal{P}$ represents the tangent plane $\Pi_{T}$ to the constraint hypersurface $S_{C}$ defined by the constraints in (13). Therefore, the iterates used by TDM and SDM essentially move on the plane $\Pi_{T}$, according to their respective approximations to the Hessian (see Fig. 14). 


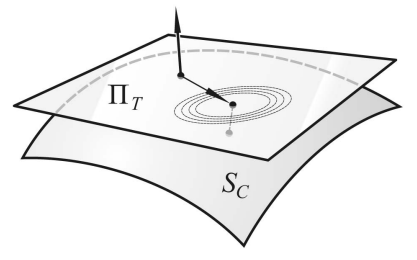

Fig. 14. Constraint surface and its tangent plane.

\section{ACKNOWLEDGMENTS}

The work described in this paper was partially supported by a grant from the Research Grants Council of the Hong Kong Special Administrative Region, China (Project No. HKU 7155/03E). Wenping Wang's work was supported by National Key Basic Research Project of China under 2004CB318000.

\section{REFERENCES}

[1] K.-S.D. Cheng, W. Wang, H. Qin, K.-Y.K. Wong, H. Yang, and Y. Liu, "Fitting Subdivision Surfaces to Unorganized Point Data Using SDM," Proc. 12th Pacific Conf. Computer Graphics and Applications (PG'04), pp. 16-24, 2004.

[2] S. Schaefer and J. Warren, "Dual Marching Cubes: Primal Contouring of Dual Grids," Proc. 12th Pacific Conf. Computer Graphics and Applications (PG '04), pp. 70-76, 2004.

[3] C. Loop, "Smooth Subdivision Surfaces Based on Triangles," MS thesis, Dept. of Math., Univ. of Utah, 1987.

[4] J. Hoschek, "Intrinsic Parameterization for Approximation," Computer Aided Geometric Design, vol. 5, pp. 27-31, 1988.

[5] G. Medioni and Y. Chen, "Object Modeling by Registration of Multiple Range Images," Image and Vision Computing, pp. 145-155, 1992.

[6] H. Pottmann and S. Leopoldseder, "A Concept for Parametric Surface Fitting Which Avoids the Parametrization Problem," Computer Aided Geometric Design, vol. 20, pp. 343-362, 2003.

[7] A. Björck, Numerical Methods for Least Squares Problems. Math. Soc. for Industrial and Applied Math., 1996.

[8] C.T. Kelley, Iterative Methods for Optimization. Soc. for Industrial and Applied Math., 1999.

[9] H. Hoppe, T. DeRose, T. Duchamp, J. McDonald, and W. Stuetzle, "Surface Reconstruction from Unorganized Points," Proc. ACM SIGGRAPH '92, pp. 71-78, 1992.

[10] M. Halstead, M. Kass, and T. DeRose, "Efficient, Fair Interpolation Using Catmull-Clark Surfaces," Proc. ACM SIGGRAPH '93, pp. 3544, 1993.

[11] W. Ma and J.P. Kruth, "Parameterization of Randomly Measured Points for Least Squares Fitting of B-Spline Curves and Surfaces," Computer-Aided Design, vol. 27, no. 9, pp. 663-675, 1995.

[12] W. Ma and N. Zhao, "Smooth Multiple B-Spline Surface Fitting with Catmull-Clark Subdivision Surfaces for Extraordinary Corner Patches," The Visual Computer, vol. 18, pp. 415-436, 2002.

[13] M. Marinov and L. Kobbelt, "Optimization Techniques for Approximation with Subdivision Surfaces," Proc. ACM Symp. Solid Modeling and Applications, pp. 1-10, 2004.

[14] H. Suzuki, S. Takeuchi, and T. Kanai, "Subdivision Surface Fitting to a Range of Points," Proc. Seventh Pacific Conf. Computer Graphics and Applications (PG'99), pp. 158-167, 1999.

[15] A. Blake and M. Isard, Active Contours. Springer, 1998.

[16] H.K. Zhao, S. Osher, B. Merriman, and M. Kang, "Implicit and Non-Parametric Shape Reconstruction from Unorganized Data Using a Variational Level Set Method," Computer Vision and Image Understanding, vol. 80, pp. 295-319, 2000.

[17] Y. Duan and H. Qin, "Intelligent Balloon: A Subdivision-Based Deformable Model for Surface Reconstruction of Arbitrary Unknown Topology," Proc. Sixth ACM Symp. Solid Modeling and Applications, pp. 47-58, 2001.

[18] N. Litke, A. Levin, and P. Schröder, "Fitting Subdivision Surfaces," Proc. 12th Ann. IEEE Visualization Conf. (VIS '01), pp. 319-324, 2001.
[19] B. Jüttler and A. Felis, "Least-Squares Fitting of Algebraic Spline Surfaces," Advances in Computational Math., vol. 17, pp. 135-152, 2002.

[20] T. Kanai, "MeshToSS: Converting Subdivision Surfaces from Dense Meshes," Proc. Vision Modeling and Visualization Conf. (VMV'01), pp. 325-332, 2001.

[21] W.-K. Jeong, I. Ivrissimtzis, and H.-P. Seidel, "Neural Meshes: Statistical Learning Based on Normals," Proc. 11th Pacific Conf. Computer Graphics and Applications (PG '03), pp. 404-408, 2003.

[22] D. Zorin, P. Schröder, A. DeRose, J. Stam, L. Kobbelt, and J. Warren, Subdivision for Modeling and Simulation, SIGGRAPH '99 Course Notes, 1999.

[23] A. Ruhe and P.-A. Wedin, "Algorithms for Separable Nonlinear Least Squares Problems," SIAM Rev., vol. 22, pp. 318-337, 1980.

[24] M. Marinov and L. Kobbelt, "Optimization Methods for Scattered Data Approximation with Subdivision Surfaces," Graphical Models, vol. 67 , no. 5, pp. 452-473, 2005.

[25] L. Ambrosio and C. Montegazza, "Curvature and Distance Function from A Manifold," J. Geometric Analysis, vol. 8, pp. 723$748,1998$.

[26] H. Pottmann and M. Hofer, "Geometry of the Squared Distance Function to Curves and Surfaces," Visualization and Math. III, pp. 223-244, Springer, 2003.

[27] H. Pottmann, S. Leopoldseder, and M. Hofer, "Approximation with Active B-spline Curves and Surfaces," Proc. 10th Pacific Conf. Computer Graphics and Applications (PG'02), pp. 8-25, 2002.

[28] W. Wang, H. Pottmann, and Y. Liu, "Fitting B-Spline Curves to Point Clouds by Squared Distance Minimization," ACM Trans. Graphics, vol. 25, no. 2, pp. 214-238, 2006.

[29] J. Stam, "Exact Evaluation of Catmull-Clark Subdivision Surfaces at Arbitrary Parameter Values," Proc. ACM SIGGRAPH '98, pp. 395-404, 1998.

[30] J. Stam, "Evaluation of Loop Subdivision Surfaces," Proc. ACM SIGGRAPH '98, on CDROM, 1998.

[31] S. Leopoldseder, H. Pottmann, and H.K. Zhao, "The $d^{2}$ Tree: A Hierarchical Representation of the Squared Distance Field," Technical Report 101, Inst. Geometry, Vienna Univ. of Technology, 2003.

[32] W. Lorensen and H. Cline, "Marching Cubes: A High Resolution 3-D Surface Construction Algorithm," Computer Graphics, vol. 21, pp. 163-169, 1987.

[33] C. Lürig, L. Kobbelt, and T. Ertl, "Hierarchical Solutions for the Deformable Surface Problem in Visualization," Graphical Models, vol. 62, pp. 2-18, 2000.

[34] V. Weiss, L. Andor, G. Renner, and T. Varady, "Advanced Surface Fitting Techniques," Computer Aided Geometric Design, vol. 19, no. 1, pp. 19-42, 2002.

[35] R.E. Bank, A.H. Sherman, and A. Weiser, "Refinement Algorithm and Data Structures for Regular Local Mesh Refinement," Scientific Computing, pp. 3-17, 1983.

[36] K. Madsen, H.B. Nielsen, and O. Tingleff, "Methods for NonLinear Least Squares Problems," Informatics and Math. Modelling, Technical Univ. of Denmark, 2004.

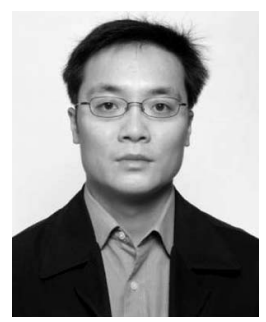

Kin-Shing D. Cheng received the BEng degree in computer engineering, with the first class honors, from the University of Hong Kong in 1997 and the MPhil and PhD degrees, both in computer graphics, from the University of Hong Kong in 2001 and 2006, respectively. His research interests include computer graphics and geometric computing. He is a member of the IEEE and the IEEE Computer Society. 


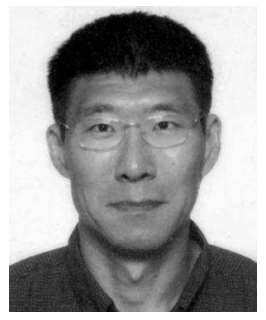

Wenping Wang received the $\mathrm{PhD}$ degree in computer science from the University of Alberta in 1992. He is an associate professor of computer science at the University of Hong Kong. He has published widely in the fields of computer graphics, geometric computing, and visualization. He is an associate editor of Computer Aided Geometric Design and has been a program cochair of several international conferences, including Geometric Modeling and Processing 2000 (GMP '00), 11th Pacific Conference on Computer Graphics and Applications 2003 (PG '03), and the ACM Symposium on Physical and Solid Modeling 2006 (SPM '06). Detailed information about him can be found from his Web site: http://www.cs.hku.hk/ wenping/.

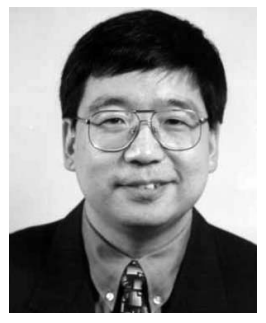

Hong Qin received the BS and MS degrees in computer science from Peking University, Beijing, and the $\mathrm{PhD}$ degree in computer science from the University of Toronto (UofT) in 1995. Currently, he is a full professor of computer science in the Department of Computer Science at the State University of New York at Stony Brook (Stony Brook University). During his years at UofT, he received a UofT Open Doctoral Fellowship. He was also a recipient of the US National Science Foundation (NSF) Faculty Early Career Development (CAREER) Award, the Honda Initiation Award, and the Alfred P. Sloan Research Fellowship by the Sloan Foundation. In 2005, he served as the general cochair for Computer Graphics International 2005 (CGl' 05). Currently, he is an associate editor for the IEEE Transactions on Visualization and Computer Graphics, and he is also on the editorial board of The Visual Computer (International Journal of Computer Graphics). In 2007, he is the conference cochair for the ACM Solid and Physical Modeling Symposium. His research interests include geometric and solid modeling, graphics, physics-based modeling and simulation, computer-aided geometric design, human-computer interaction, visualization, and scientific computing. He is a member of the IEEE. http://www.cs.sunysb.edu/ qin.

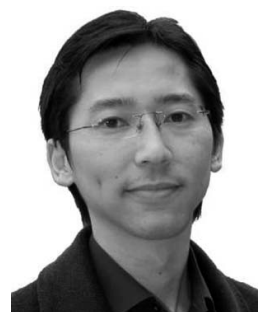

Kwan-Yee K. Wong received the BEng degree in computer engineering, with first class honors, from the Chinese University of Hong Kong in 1998. From 1998 to 2001, he studied in the United Kingdom at the University of Cambridge, and he received the MPhil and PhD degrees, both in computer vision, from the University of Cambridge in 2000 and 2001, respectively. Since 2001, he has been with the Department of Computer Science at the University of Hong Kong, where he is now an assistant professor. His research interests are in computer vision and image processing, including camera calibration, motion tracking, model reconstruction and representation, and motion estimation from image sequences. He is a member of the IEEE.

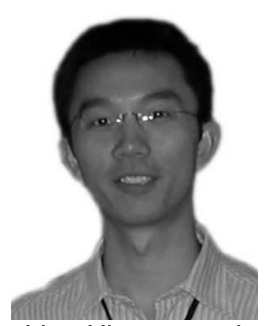

Huaiping Yang received the BE degree in hydraulic engineering and the $\mathrm{PhD}$ degree in computer science from Tsinghua University, China, in 1998 and 2004, respectively. In 2004, he did a one-year postdoctorate program in the Computer Graphics Group at the University of Hong Kong. In 2005, he started a postdoctorate program at Johannes Kepler University Linz, Austria, in the field of applied geometry, funded by a Marie Curie Incoming International Fellowship. His research interests include geometric modeling, computer graphics, and scientific visualization.

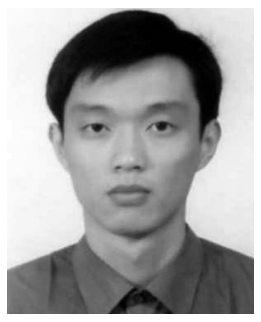

Yang Liu received the BS and MS degrees in mathematics from the University of Science and Technology of China in 2000 and 2003, respectively. He is a PhD student in the Computer Science Department at the University of Hong Kong. His research interests include computeraided geometric design, computer graphics, and computational algebraic geometry. Detailed information about him can be found from his Web site: http://www.cs.hku.hk/ yliu/

$\triangleright$ For more information on this or any other computing topic, please visit our Digital Library at www.computer.org/publications/dlib. 